'It is unlikely that there will be a

single effect of bilingualism'

-Kroll \& Chiarello, 2016, p. 347

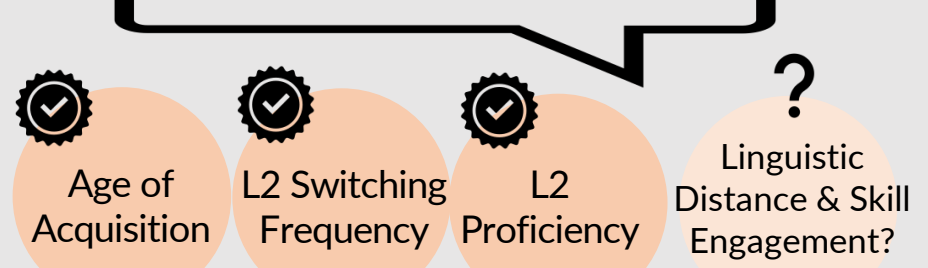

$\mathrm{L}$

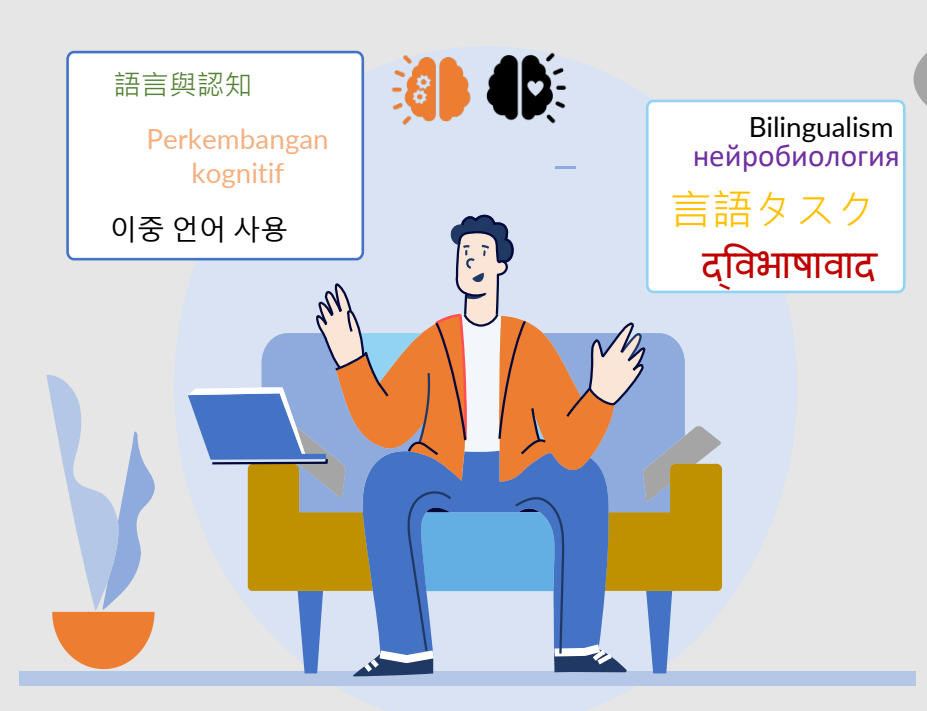

\title{
Engagement
}

Under the umbrella of typological distance, the type of skill(s) that a bilingual uses in their $L 2$ may have an influence on cognitive demand

Imagine the different levels of inhibitory control needed in these scenarios:

Reading in a typologically similar L1-L2 pair (e.g.,

Spanish-Portuguese) vs. Producing in a typologically similar L1-L2 pair

Or, imagine the different types of memory needed for the following scenarios:

Recognizing the logographic characters of a distant L2 (e.g., Mandarin characters) vs. writing them out by memory

Neuroimaging research thus far gives insight into how linguistic the specific designs of a

a

n 'L1-L2 Typological Distance' is not welldefined in bilingualism scholarship

g

This definition is crucial because

U languages can differ exponentially

in various linguistic dimensions. The

a processing of these dimensions can

take up different levels of effort

g depending on the individual's

background languages and

e perceived L1-L2 distance (i.e., psychotypology).

language may activate different parts of the brain. This has implications for processing across various dimensions of typological distance:

Complexity of
grammatical grammatica De Martino et al, 2020

\section{Dimensions of Typological Distance}

Lexical status orthographic similarity

$$
\text { Baeck et al., } 2015
$$

Logographic vs. alphabetic scripts coderre et al., 2008

\section{Neuroscience}

The fundamental connection between bilingual neural activity and neurostructural changes is not yet strongly confirmed

Scientists are still unsure whether repeated engagement with a brain function in bilingual activity necessarily leads to neuroanatomical changes. This foundation is key for further investigations into the impact of L1-L2 typological distance in the bilingual brain. 